\title{
Molecular diagnosis of maturity-onset diabetes of the young in a cohort of Chinese children
}

\begin{abstract}
Objective: The purpose of this study was to investigate the molecular basis of maturity-onset diabetes of the young (MODY) by whole-exome sequencing (WES) and estimate the frequency and describe the clinical characteristics of MODY in southern China. Methods: Genetic analysis was performed in 42 patients with MODY aged 1 month to 18 years among a cohort of 759 patients with diabetes, identified with the following four clinical criteria: age of diagnosis $\leq 18$ years; negative pancreatic autoantibodies; family history of diabetes; or persistently detectable C-peptide; or diabetes associated with extrapancreatic features. GCK gene mutations were first screened by Sanger sequencing. GCK mutation-negative patients were further analyzed by WES. Results: Mutations were identified in 24 patients: 20 mutations in GCK, 1 in HNF4A, 1 in INS, 1 in ABCC8, and a 17q12 microdeletion. Four previously unpublished novel GCK mutations: c.1108G>C in exon 9, and c.1339C>T, c.1288_1290delCTG, and c.1340_1343delGGGGinsCTGGTCT in exon 10 were detected. WES identified a novel missense mutation c.311A $>\mathrm{G}$ in exon 3 in the INS gene, and copy number variation analysis detected a $1.4 \mathrm{Mb}$ microdeletion in the long arm of the chromosome $17 q 12$ region. Compared with mutation-negative subjects, the mutation-positive subjects had lower hemoglobin A1c and initial blood glucose levels. Conclusions: Most MODY cases in this study were due to GCK mutations, which is in contrast to previous reports in Chinese patients. Diabetes associated with extrapancreatic features should be a clinical criterion for MODY genetic analysis. Mutational analysis by WES provided a precise diagnosis of MODY subtypes. Moreover, WES can be useful for detecting large deletions in coding regions in addition to point mutations.
\end{abstract}

